\title{
Development of Cortical Networks under Continuous Stimulation
}

\author{
Ophir Orenstein ${ }^{1,2}$ and Hanna Keren ${ }^{1,2 *}$ \\ ${ }^{1}$ Network Biology Research Laboratory, Electrical Engineering, Technion - Israel Institute of Technology, Haifa, Israel, \\ ${ }^{2}$ Department of Physiology, Biophysics and Systems Biology, Technion - Israel Institute of Technology, Haifa, Israel
}

Keywords: neural networks, development, stimulation, synchronization, cultures

\section{INTRODUCTION}

Neural networks develop naturally under continuous reciprocal interaction with the environment, which results in early life experiences having an immense impact on adult capacities to function and adapt to the environment (Vygotsky, 1978; Black et al., 1987; Greenough et al., 1987; Sur et al., 1988; Pallas et al., 1990; Jones and Greenough, 1996; Markham and Greenough, 2004; Marom, 2015). It is well established, for example, that sensory inputs during development play an essential role in shaping brain circuitry and neuronal connectivity, as was shown by the classical studies of Hubel and Wiesel in which sensory deprivation enhanced fundamental alterations of primary sensory areas (Hubel and Wiesel, 1977; Hubel et al., 1977; Greenough et al., 1987; Sur et al., 1988). However, the underlying neural principles and mechanisms which impact such developmental interactions remain largely unknown. This state-of-the-art environment-development problem, calls for controlled, multi-level experimental access to neural networks development over a wide range of temporal and spatial scales. The major aim of this work, therefore, is to provide an experimental database for studying how external inputs encountered during development can affect the mature aspects of neuronal network activity-implemented in neural networks developing in-vitro.

Cultures of cortical networks comprise a relevant experimental model for characterizing development of neural activity, without being affected by predefined constraints and intervening processes (Katz, 1993; Wong, 1993; Marom and Shahaf, 2002). The developmental stages and their corresponding time frames are surprisingly similar to those observed in vivo. When a population of cortical neurons is extracted from a rat newborn and allowed to develop for a couple of weeks outside the brain, it forms a network that exhibits complex spontaneous activity. During this process, uncorrelated firing appears at the end of the first week in culture, then evolving to synchronous activations observed as early as 9-12 days in-vitro (Gross, 1979; Habets et al., 1987; Stenger and McKenna, 1994; Kamioka et al., 1996; Corner et al., 2002; Marom and Shahaf, 2002; Morin et al., 2005; Eytan and Marom, 2006; Wagenaar et al., 2006). Synchronous events frequency increase to an average rate of ca. $0.1 \mathrm{~Hz}$, in dependence on a rapid growth in networks connectivity, both in terms of number of connections and connection length, between 5 and 10 days in-vitro (Maeda et al., 1995; Marom and Shahaf, 2002; van Pelt et al., 2005; Wagenaar et al., 2006; Napoli et al., 2014).

By applying electric stimulation to these networks, it is possible to evoke a synchronized response that propagates across extended parts of the network (Maeda et al., 1995; Pinato et al., 1999; Jimbo et al., 2000; Marom and Shahaf, 2002; Wagenaar et al., 2004). Such stimulation has been shown to simulate modulatory effects of sensory inputs, as inducing neural plasticity and changes in network connectivity (Jimbo et al., 1998, 1999; Maeda et al., 1998; Tateno and Jimbo, 1999; Rolston et al., 2007; Bologna et al., 2010; Keren and Marom, 2014). This model provides a 
well-controlled setting for studying impacts of external inputs during development, as addressed by previous studies in which activity was sampled along development in-vitro following sparse stimulation phases (Wagenaar et al., 2006; Bologna et al., 2010).

This work, extends these studies in the direction of continuous recording and stimulation of cortical networks in the course of development, as of the second day in-vitro. The experimental database is comprised of six networks developing while exposed to continuous stimulation, as well as an additional two nonstimulated networks, similarly monitored. Stimulation was applied alternately from two spatially distinct sources with a uniform distribution of inter-stimulus-intervals ranging from 6 to $14 \mathrm{~s}$. Ultra-slow perfusion of culture medium was used to ensure this ongoing long-range experimental setting. Examples given in Figure 1, present extracts of recorded activities during the ninth day in-vitro of two non-stimulated networks (A) and two continuously stimulated ones (B). Note that in four of the six stimulated networks, parts of the sampled region could not be detected to a certain extent, possibly due to deficiencies in technical monitoring, thus are absent from synchronized activity (synchronization properties, however, are still apparent and measurable in these cases, as exemplified in Figure 1B, left).

We chose to address this data using synchronization as a system variable, relevant for neural network function and activity and driven by external inputs. The development of synchronization in particular, has been suggested as the underling process for the fine-tuning of mature, functional neural circuits (Yuste et al., 1992; Katz and Shatz, 1996; Zhang and Poo, 2001; Leinekugel et al., 2002; Corlew et al., 2004; Khazipov et al., 2004; Kilb et al., 2011). Our experiments outline the gradual emergence of network synchronization between days
6 and 9, as shown in Figure 2A. Moreover, in this context, functionality can be approached through the capacity to classify (i.e., discriminate between) different inputs, a primitive that stands at the basis of cognitive capabilities. The development of efficient classification between two stimulation sources, estimated by recruitment orders of spike representations, is described in Figure 2B (left), as is its possible dependence on synchronization rate (example given in Figure 2B, right). Exposure to stimulation during development, is suggested to impact networks' mature activity, as demonstrated by a potential increase in synchronous activity properties of relative amplitude and duration (evident in Figure 1B), as well as by an increase of active electrodes participation during synchronization (see Figure 2C, reflecting the count of spikes during synchronization by each electrode). It should be noted that each network was cultured in a separate batch, repeating the same procedure using different animals.

Fruitful directions for research might include questions as diverse as the dependence of mature propagation orders of neurons across the network on initial activity-connectivity profiles (e.g., we found that units generating early activities are recruited later across the network in mature synchrony propagation, reflecting early activation convergence to a "follower" type of activation) and aspects of activity rhythms occurring along a scale of hours to days. Experimental extensions toward full reciprocal interaction with the environment by closing the loop on a key measure that is relevant for function and that is affected by external inputs, could modify the experienced environment and enhance underlying alterations of activity occurring during development (Shahaf and Marom, 2001; Wagenaar and Potter, 2004; Wagenaar et al., 2005; Arsiero et al., 2007; Rolston et al., 2010; Keren and Marom, 2014). Overall, we

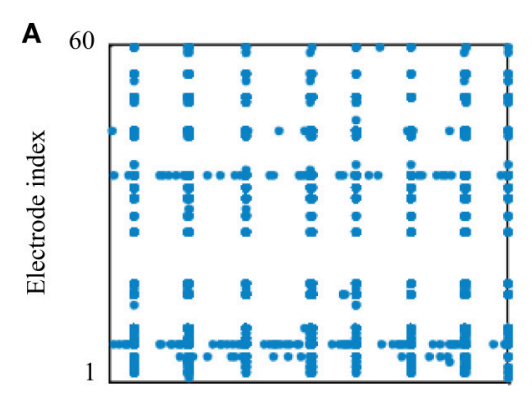

B

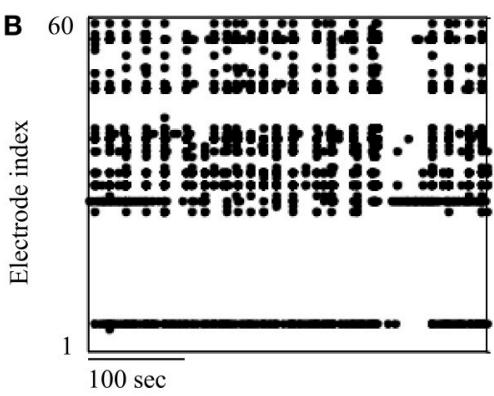

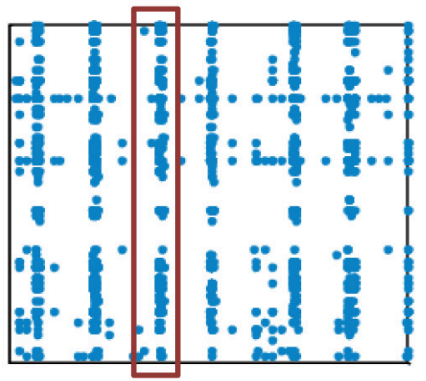

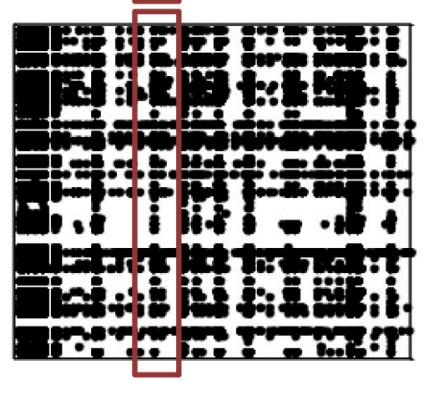

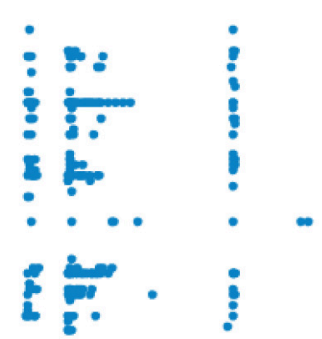

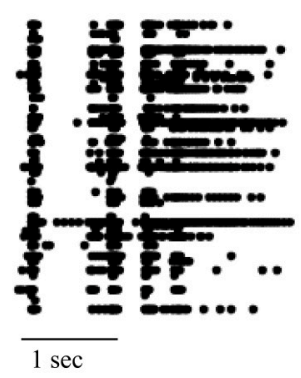

FIGURE 1 | Neural networks development data. Segments of activity recorded during the ninth day in-vitro (DIV) are presented in (A) for two non-stimulated networks and in (B) for two networks exposed to continuous stimulation. Each point depicts a single spike detected in one of the electrodes (indexed in the vertical axis); the right-hand panel shows the boxed synchronous event at higher temporal resolution. 
believe that the provided database and proposed interpretations have the potential to contribute insights regarding environmental interaction with the course of neural development.

\section{MATERIALS AND METHODS}

\subsection{Cell Preparation}

Cortical neurons were obtained from newborn rats (SpragueDawley) within $24 \mathrm{~h}$ after birth using mechanical and enzymatic procedures described in earlier studies (Marom and Shahaf, 2002). Rats were anesthetized by $\mathrm{CO}_{2}$ inhalation according to protocols approved by the Technion's ethics committee. The neurons were plated directly onto a substrate-integrated multi electrode array and allowed to develop into functionally and structurally mature networks over a period of $2-3$ weeks, while being continuously recorded and stimulated. The number of plated neurons was of the order of 450,000, covering an area of about $380 \mathrm{~mm}^{2}$. The preparations were perfused continuously (ultra slow perfusion with an exchange rate of about $0.06 \mathrm{ml} / \mathrm{h}$ ) with MEM supplemented with heat-inactivated horse serum (5\%), glutamine $(0.5 \mathrm{mM})$, glucose $(20 \mathrm{mM})$, and gentamycin (10 $\mu \mathrm{g} / \mathrm{ml}$ ), and maintained in an atmosphere of $37^{\circ} \mathrm{C}, 5 \% \mathrm{CO}_{2}$ and $95 \%$ air.

\subsection{Electrophysiology}

An array of Ti/Au extracellular electrodes, $30 \mu \mathrm{m}$ in diameter, spaced $500 \mu \mathrm{m}$ from each other and located in the center, was used (MultiChannelSystems, Reutlingen, Germany). A commercial amplifier (MEA-1060-inv-BC, MCS, Reutlingen, Germany) with frequency limits of $150-3000 \mathrm{~Hz}$ and a gain of x1024 was obtaining data. Data was digitized using data acquisition board (PD2-MF-64-3M/12H, UEI, Walpole, MA, USA). Each channel was sampled at a frequency of 16 $\mathrm{kHz}$. The insulation layer (silicon nitride) was pre-treated with polyethyleneimine (Sigma, $0.01 \%$ in $0.1 \mathrm{M}$ Borate buffer solution). Data acquired was analyzed using Matlab (Mathworks, Natick, MA, USA).

\subsection{Experimental Procedure}

Following cell plating, networks were kept in an incubator with controlled conditions as described above for $24-48 \mathrm{~h}$, and then placed on the recording setup for the entire duration of the experiment (9 DIV being the shortest experiment and 43 DIV the longest). Six networks were stimulated via two alternating, spatially distant electrodes (29 and 49 , in this order), with interstimulus-intervals ranging between 6 and $14 \mathrm{~s}$ and uniformly distributed $(0.12 \mathrm{~Hz} \pm 0.04)$. Each recording day included an hour with no stimulation, when spontaneous activity data was obtained. Two experiments depict spontaneous activity only, recorded along the development (experiments \#1 and \#8). In all cases, recording was stopped once every 3 days for up to $30 \mathrm{~min}$ for maintenance of the perfusion system.

\subsection{Analyses}

Action potentials were detected by threshold crossing which is defined separately for each of the recording channels at the beginning of an experiment $(6 \times$ standard deviation of a 2
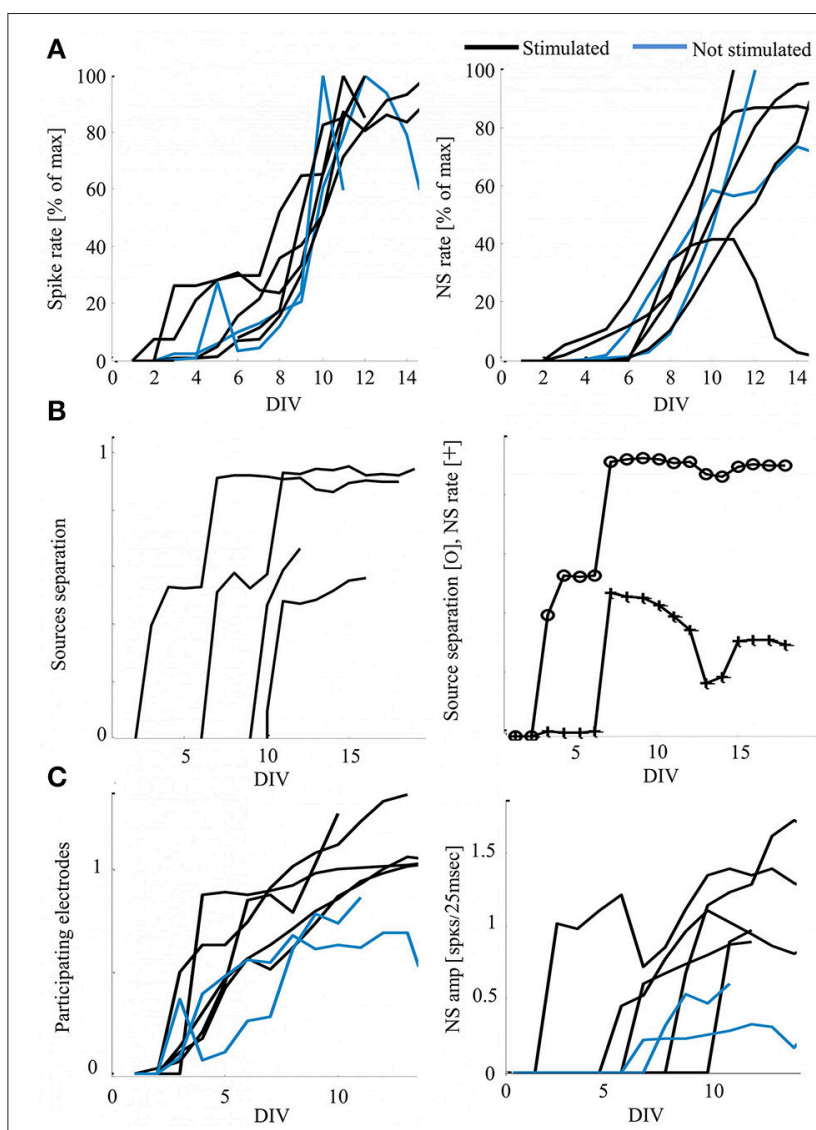

FIGURE 2 | Development of activity-functionality measures and possible impacts of stimulation. (A) Development of activity rate measures, calculated as the mean value per day in-vitro (DIV) normalized to the individual maximal value for presentation clarity $(\mathrm{Hz})$. Each curve represents a single network, color-coded according to the developmental conditions. (B) Examples of stimulated networks demonstrating increasing distance between recruitment orders evoked by the two stimulation sources. Order vectors are comprised of the first spikes of the first five electrodes; the distance is calculated as the average of a pair-wise distance matrix using Levenshtein Edit Distance metric (Shahaf et al., 2008). Right: an example of the possible correspondence between the classification of stimulation sources (distance) and the response rate, scaled for presentation clarity $(C C=0.83)$. (C) Stimulated networks (black) show increased number of participating electrodes per day in-vitro (left, mean values of 0.51 and 0.7 for non-stimulated and exposed to stimulation networks, respectively, with $p<$ 0.005 by $t$-test) and synchronous events amplitude (right, mean values of 0.26 and 0.62 for non-stimulated and exposed to stimulation networks, respectively, with $p<0.005$ by $t$-test). Normalization across networks is to the number of electrodes active in each network during the last day of recording (active is considered as $>5$ spikes).

$\mathrm{s}$ voltage trace). A refractory period of $6 \mathrm{~ms}$ was considered. Electrical activity detected often originates from several sources, typically 3-2 neurons, as each recording electrode is surrounded by several cell bodies. Detection of synchronous events was performed on-line by threshold crossing of summed action potentials within $25 \mathrm{~ms}$. Exact threshold was determined relative to $25 \%$ of the active electrodes (typically the value of 20 action potentials). 


\subsection{Overview and Format of the Database}

The database can be accessed through the following link: https:// figshare.com/s/5cff3bb4e25b814c1459. It contains separate datasets ("projects") for each of the experiments (files within are numbered accordingly, from 1 to 8 ). Each dataset comprises text files numbered by their recording order. The text files can be opened using various interfaces, such as text editors, Excel, Matlab or Mathematica and include a table of time stamps (in seconds) of all spikes (in the first column); the corresponding electrode indexes for each of the spikes (row-wise in the second column); and the shape of each spike (as of the third column; Each column represents a sample unit equals to $0.0625 \mathrm{msec}$ and voltage units are of $0.42 \mu \mathrm{V}$ ). Loading these files to Matlab might provide two variables: textdata-with the two leftmost columns representing the spike time stamps and electrode index, respectively; and data, which describes the spike shape corresponding to each of the spikes indicated by the file textdata (note that the first two rows of textdata are excluded).

Beyond the 60 electrodes of the recording array, index 61 -represents time stamps of stimulation, and index 64 represents the detection of a synchronous event (the time of threshold crossing in an on-line detection). Electrodes 63 and 62 depict internal blanking signals and should be omitted (typically, the first two spikes-rows, of each data file).

The time stamps across consecutive files are continuous up to the reset of recording for maintenance, which occurred about every 3 days (which caused a restart of time stamps to zero). A continuous activity times vector can be generated by adding the last time stamp prior to the reset to all the consecutive times of the following data files. The stimulation

\section{REFERENCES}

Arsiero, M., Lüescher, H. R., and Giugliano, M. (2007). Real-time closed-loop electrophysiology: towards new frontiers in in vitro investigations in the neurosciences. Arch. Ital. Biol. 145, 193-209.

Black, J. E., Sirevaag, A. M., and Greenough, W. T. (1987). Complex experience promotes capillary formation in young rat visual cortex. Neurosci. Lett. 83, 351-355. doi: 10.1016/0304-3940(87)90113-3

Bologna, L. L., Nieus, T., Tedesco, M., Chiappalone, M., Benfenati, F., and Martinoia, S. (2010). Low-frequency stimulation enhances burst activity in cortical cultures during development. Neuroscience 165, 692-704. doi: 10.1016/j.neuroscience.2009.11.018

Corlew, R., Bosma, M. M., and Moody, W. J. (2004). Spontaneous, synchronous electrical activity in neonatal mouse cortical neurones. J. Physiol. 560(Pt 2), 377-390. doi: 10.1113/jphysiol.2004.071621

Corner, M. A., van Pelt, J., Wolters, P. S., Baker, R. E., and Nuytinck, R. H. (2002). Physiological effects of sustained blockade of excitatory synaptic transmission on spontaneously active developing neuronal networksan inquiry into the reciprocal linkage between intrinsic biorhythms and neuroplasticity in early ontogeny. Neurosci. Biobehav. Rev. 26, 127-185. doi: 10.1016/S0149-7634(01)00062-8

Eytan, D., and Marom, S. (2006). Dynamics and effective topology underlying synchronization in networks of cortical neurons. J. Neurosci. 26, 8465-8476. doi: 10.1523/JNEUROSCI.1627-06.2006

Greenough, W. T., Black, J. E., and Wallace, C. S. (1987). Experience and brain development. Child Dev. 58, 539-559. doi: 10.2307/1130197 electrode index (29 and 49, alternating) is given in a separate file named StimulusIndex. This file comprises 25,920 stimuli and was reused at each reset of recording. An hour of no stimulation was integrated daily for the stimulated networks, indicated by an index of " 0 " in the StimulusIndex file (as of stimulation 25,559). The respective stimulation times are given in the file StimulusTimes. For simplification of analyses, an additional file named Days provides the organization of all experiments files by the recording day in-vitro (an Excel file that presents the range of file numbers belonging to a day, with days indicated by the row number and each experiment organized in a separate sheet). The spatial layout of all electrodes is provided in a file named Array, in which electrode indexes are schemed by their spatial location.

Through-out the recordings, artifact synchronous activities may occur, characterized by an immediate precise synchrony across the entire culture, a typical event in such experimental systems (possibly due to a drop of vaporized liquid). These events can simply be excluded from analyses by detecting the occurrence of a synchronous event in all electrodes in a time window as short as a couple of milliseconds.

\section{AUTHOR CONTRIBUTIONS}

OO performed the experiments, initial analyses, and created the database, HK performed the analyses and wrote the manuscript.

\section{ACKNOWLEDGMENTS}

The authors thank Shimon Marom for his much valuable advice and guidance.
Gross, G. W. (1979). Simultaneous single unit recording in vitro with a photoetched laser deinsulated gold multimicroelectrode surface. IEEE Trans. Biomed. Eng. 26, 273-279. doi: 10.1109/TBME.1979.326402

Habets, A., Van Dongen, A., Van Huizen, F., and Corner, M. (1987). Spontaneous neuronal firing patterns in fetal rat cortical networks during development in vitro: a quantitative analysis. Exp. Brain Res. 69, 43-52. doi: 10.1007/BF00247027

Hubel, D. H., and Wiesel, T. N. (1977). Ferrier lecture. Functional architecture of macaque monkey visual cortex. Proc. R. Soc. Lond. B Biol. Sci. 198, 1-59. doi: 10.1098/rspb.1977.0085

Hubel, D. H., Wiesel, T. N., and Stryker, M. P. (1977). Orientation columns in macaque monkey visual cortex demonstrated by the 2-deoxyglucose autoradiographic technique. Nature 269, 328-330. doi: 10.1038/269 $328 \mathrm{a} 0$

Jimbo, Y., Kawana, A., Parodi, P., and Torre, V. (2000). The dynamics of a neuronal culture of dissociated cortical neurons of neonatal rats. Biol. Cybern. 83, 1-20. doi: 10.1007/PL00007970

Jimbo, Y., Robinson, H., and Kawana, A. (1998). Strengthening of synchronized activity by tetanic stimulation in cortical cultures: application of planar electrode arrays. IEEE Trans. Biomed. Eng. 45, 1297-1304. doi: $10.1109 / 10.725326$

Jimbo, Y., Tateno, T., and Robinson, H. (1999). Simultaneous induction of pathway-specific potentiation and depression in networks of cortical neurons. Biophys. J. 76, 670-678. doi: 10.1016/S0006-3495(99)77234-6

Jones, T. A., and Greenough, W. T. (1996). Ultrastructural evidence for increased contact between astrocytes and synapses in rats reared in a 
complex environment. Neurobiol. Learn. Mem. 65, 48-56. doi: 10.1006/nlme. 1996.0005

Kamioka, H., Maeda, E., Jimbo, Y., Robinson, H. P., and Kawana, A. (1996). Spontaneous periodic synchronized bursting during formation of mature patterns of connections in cortical cultures. Neurosci. Lett. 206, 109-112. doi: 10.1016/S0304-3940(96)12448-4

Katz, L. C. (1993). Coordinate activity in retinal and cortical development. Curr. Opin. Neurobiol. 3, 93-99. doi: 10.1016/0959-4388(93)90041-V

Katz, L. C., and Shatz, C. J. (1996). Synaptic activity and the construction of cortical circuits. Science 274, 1133-1138. doi: 10.1126/science.274.5290.1133

Keren, H., and Marom, S. (2014). Controlling neural network responsiveness: tradeoffs and constraints. Front. Neuroeng. 7:11. doi: 10.3389/fneng.2014.00011

Khazipov, R., Khalilov, I., Tyzio, R., Morozova, E., Ben-Ari, Y., and Holmes, G. L. (2004). Developmental changes in gabaergic actions and seizure susceptibility in the rat hippocampus. Eur. J. Neurosci. 19, 590-600. doi: 10.1111/j.0953-816X.2003.03152.x

Kilb, W., Kirischuk, S., and Luhmann, H. J. (2011). Electrical activity patterns and the functional maturation of the neocortex. Eur. J. Neurosci. 34, 1677-1686. doi: 10.1111/j.1460-9568.2011.07878.x

Leinekugel, X., Khazipov, R., Cannon, R., Hirase, H., Ben-Ari, Y., and Buzsáki, G. (2002). Correlated bursts of activity in the neonatal hippocampus in vivo. Science 296, 2049-2052. doi: 10.1126/science.1071111

Maeda, E., Kuroda, Y., Robinson, H. P., and Kawana, A. (1998). Modification of parallel activity elicited by propagating bursts in developing networks of rat cortical neurones. Eur. J. Neurosci. 10, 488-496. doi: 10.1046/j.1460-9568.1998.00062.x

Maeda, E., Robinson, H. P., and Kawana, A. (1995). The mechanisms of generation and propagation of synchronized bursting in developing networks of cortical neurons. J. Neurosci. 15, 6834-6845.

Markham, J. A., and Greenough, W. T. (2004). Experience-driven brain plasticity: beyond the synapse. Neuron Glia Biol. 1, 351-363. doi: 10.1017/S1740925X05000219

Marom, S. (2015). Science, Psychoanalysis, and the Brain: Space for Dialogue. Cambridge University Press.

Marom, S., and Shahaf, G. (2002). Development, learning and memory in large random networks of cortical neurons: lessons beyond anatomy. Q. Rev. Biophys. 35, 63-87. doi: 10.1017/S0033583501003742

Morin, F. O., Takamura, Y., and Tamiya, E. (2005). Investigating neuronal activity with planar microelectrode arrays: achievements and new perspectives. J. Biosci. Bioeng. 100, 131-143. doi: 10.1263/jbb.100.131

Napoli, A., Xie, J., and Obeid, I. (2014). Understanding the temporal evolution of neuronal connectivity in cultured networks using statistical analysis. BMC Neurosci. 15:17. doi: 10.1186/1471-2202-15-17

Pallas, S. L., Roe, A. W., and Sur, M. (1990). Visual projections induced into the auditory pathway of ferrets. i. novel inputs to primary auditory cortex (ai) from the lp/pulvinar complex and the topography of the mgn-ai projection. J. Comp. Neurol. 298, 50-68. doi: 10.1002/cne.902980105

Pinato, G., Parodi, P., Bisso, A., Macri, D., Kawana, A., Jimbo, Y., and Torre, V. (1999). Properties of the evoked spatio-temporal electrical activity in neuronal assemblies. Rev. Neurosci. 10, 279-290. doi: 10.1515/REVNEURO.1999.10.3-4.279

Rolston, J. D., Gross, R. E., and Potter, S. M. (2010). Closed-loop, open-source electrophysiology. Front. Neurosci. 4:31. doi: 10.3389/fnins.2010.00031
Rolston, J. D., Wagenaar, D. A., and Potter, S. M. (2007). Precisely timed spatiotemporal patterns of neural activity in dissociated cortical cultures. Neuroscience 148, 294-303. doi: 10.1016/j.neuroscience.2007. 05.025

Shahaf, G., Eytan, D., Gal, A., Kermany, E., Lyakhov, V., Zrenner, C., et al. (2008). Order-based representation in random networks of cortical neurons. PLoS Comput. Biol. 4:e1000228. doi: 10.1371/journal.pcbi.1000228

Shahaf, G., and Marom, S. (2001). Learning in networks of cortical neurons. J. Neurosci. 21, 8782-8788.

Stenger, D., and McKenna, T. (1994). Enabling Technologies for Cultured Neural Networks. Academic Press.

Sur, M., Garraghty, P. E., and Roe, A. W. (1988). Experimentally induced visual projections into auditory thalamus and cortex. Science 242, 1437-1441. doi: $10.1126 /$ science. 2462279

Tateno, T., and Jimbo, Y. (1999). Activity-dependent enhancement in the reliability of correlated spike timings in cultured cortical neurons. Biol. Cybern. 80, 45-55. doi: $10.1007 / \mathrm{s} 004220050503$

van Pelt, J., Vajda, I., Wolters, P. S., Corner, M. A., and Ramakers, G. J. (2005). Dynamics and plasticity in developing neuronal networks in vitro. Prog. Brain Res. 147, 173-88. doi: 10.1016/S0079-6123(04)47013-7

Vygotsky, L. S. (1978). Mind in Society: The Development of Higher Psychological Processes. Cambridge, MA: Harvard University Press.

Wagenaar, D. A., Madhavan, R., Pine, J., and Potter, S. M. (2005). Controlling bursting in cortical cultures with closed-loop multi-electrode stimulation. $J$. Neurosci. 25, 680-688. doi: 10.1523/JNEUROSCI.4209-04.2005

Wagenaar, D. A., Nadasdy, Z., and Potter, S. M. (2006). Persistent dynamic attractors in activity patterns of cultured neuronal networks. Phys. Rev. E Stat. Nonlin. Soft Matter Phys. 73:051907. doi: 10.1103/PhysRevE.73. 051907

Wagenaar, D. A., Pine, J., and Potter, S. M. (2004). Effective parameters for stimulation of dissociated cultures using multi-electrode arrays. J. Neurosci. Methods 138, 27-37. doi: 10.1016/j.jneumeth.2004.03.005

Wagenaar, D. A., and Potter, S. M. (2004). A versatile all-channel stimulator for electrode arrays, with real-time control. J. Neural Eng. 1, 39-45. doi: 10.1088/1741-2560/1/1/006

Wong, R. O. (1993). The role of spatio-temporal firing patterns in neuronal development of sensory systems. Curr. Opin. Neurobiol. 3, 595-601. doi: 10.1016/0959-4388(93)90061-3

Yuste, R., Peinado, A., and Katz, L. C. (1992). Neuronal domains in developing neocortex. Science 257, 665-669. doi: 10.1126/science.1496379

Zhang, L., and Poo, M. (2001). Electrical activity and development of neural circuits. Nat. Neurosci. 4(Suppl), 1207-1214. doi: 10.1038/nn753

Conflict of Interest Statement: The authors declare that the research was conducted in the absence of any commercial or financial relationships that could be construed as a potential conflict of interest.

Copyright (c) 2017 Orenstein and Keren. This is an open-access article distributed under the terms of the Creative Commons Attribution License (CC BY). The use, distribution or reproduction in other forums is permitted, provided the original author(s) or licensor are credited and that the original publication in this journal is cited, in accordance with accepted academic practice. No use, distribution or reproduction is permitted which does not comply with these terms. 